created, addressing knowledge gaps highlighted by the surveys; doctors are given access to this in advance of their face to face sessions. The surveys will be repeated in future to determine whether this measure has helped ease the transition of new doctors joining the intensive care units.

\section{G29(P) QUANTIFYING PAEDIATRIC HIGH DEPENDENCY CARE: DOES THE PAEDIATRIC CRITICAL CARE MINIMUM DATASET ACCURATELY CAPTURE WORKLOAD?}

J Campion-Smith, S Timperley, S Edees. Paediatrics, Royal Berkshire Hospital, Reading, UK

\subsection{6/archdischild-2018-rcpch.28}

Aims The structure of paediatric critical care (PCC) in the UK is under review. ${ }^{1}$ This study aimed to evaluate the paediatric high dependency unit (PHDU) workload at our district general hospital, mapping it to the Paediatric Critical Care Minimum Dataset (PCCMDS).

Methods The demographics, diagnostic category, interventions required and outcomes of all PHDU admissions were recorded prospectively over a 4 month period (winter 2016-2017). Demographic data was compared to previous local PHDU audits and the intensity of interventions each patient received was assessed using the PCCMDS.

Results 105 patients were admitted with a mean age of 4.9 years (median 2.6 years, range 12 days-16 years 3 months). Age distribution was similar to previous years but number of admissions has greatly increased $(n=34$ in 2000-2001). Respiratory admissions are increasingly common $(33 \%$ in $2000-$ 2001 vs $55 \%$ in 2016-2007). Mean length of stay was 1.6 days (range 3 hours-12 days). 90 patients were discharged to the ward or home and 9 transferred to a tertiary centre (6 to paediatric intensive care, 1 to PHDU, 2 to wards). Outcome data was not recorded for 6 . Regarding intensity of care: 66 patients received PCCMDS basic interventions; with 2 further patients meeting the suggested new criteria. ${ }^{1} 11$ patients had intermediate interventions (8 also had basic interventions). 34 patients received no PCCMDS interventions. These were mainly neurology $(n=13)$ and poisoning $(n=9)$ admissions. 3 of this sub-group received volume resuscitation and 4 were transferred to a tertiary centre.

Conclusions Paediatric high dependency workload is increasing, particularly respiratory admissions. The PCCMDS improves understanding of the PHDU workload and will enable comparison of work between units. However 32\% of patients admitted to our PHDU received no PCCMDS interventions prompting review of our PHDU admission criteria and highlighting that some interventions are not recognised by the PCCMDS (including volume resuscitation and observation after prolonged seizures) leading to potential underestimation of workload. We recommend further modification of the PCCMDS to account for this.

\section{REFERENCE}

1. High Dependency Care for Children - Time To Move On, RCPCH, 2014.

\section{G30(P) BULIMIA NERVOSA - THE SILENT KILLER - A CASE REPORT FROM PAEDIATRIC INTENSIVE CARE}

SK Martin, J Freeman, R Ardill. Paediatric Intensive Care, NHS Lothian, Edinburgh, UK

10.1136/archdischild-2018-rcpch.29
A 14 year old boy was admitted to the paediatric intensive care unit following an out of hospital cardiac arrest. He was found to be in Ventricular Tachycardia by the ambulance service and one DC shock was administered at the scene and cardio-version achieved.

On arrival to hospital a capillary blood gas revealed a severe hypocholaraemic hypokalaemic metabolic alkalosis. PH 7.89, PCO2 2.4, NA+126, $\mathrm{K}+1.5, \mathrm{Cl} 22$. $\mathrm{BE}+\mathrm{ve} 18$. Bloods on admission showed an acute kidney injury creatinine 129, urea 8.0. The patient was gradually hydrated and electrolyte disturbance was corrected over 48 hours.

An ECG on arrival to accident and emergency showed a prolonged QTC of $675 \mathrm{~ms}$. An echocardiogram confirmed that the heart was structurally normal. The patient was unresponsive on admission to hospital (GCS 4) he was therefore intubated and ventilated and transferred to PICU.

The differential diagnosis in this case was broad and included Bartters syndrome, Gitelmans syndrome, gastric outlet obstruction, HNF-1 beta, renal, metabolic and endocrine causes.

The patient was extubated twenty- four hours following admission. He was assessed by the neurology team and he was found to have no residual neurological deficit. On waking the patient disclosed that he had struggled with body image and perceptions about his weight for the last 3 years. His enteral intake had been good but he would induce vomiting after every meal. He denied laxative use but admitted to drinking highly caffeinated drinks to keep his energy levels up. The combination of severe hypokalaemia $<1$ and high caffeine intake may have lead to Torsaides de pointes and ultimately cardiac arrest.

A history of chronic vomiting fits with the profound hypocholaraemic hypokalaemic metabolic acidosis observed on admission. Metabolic and genetic investigations were normal. Interestingly the blood gas taken in accident and emergency was taken thirty minutes after a return of spontaneous circulation had been acheived. Therefore the patients $\mathrm{PH}$ and potassium levels may have been higher and lower respectively at the time of cardiac arrest. Theoretically the patient will have started to correct his alkalosis and profound hypokalaemia as cardiac arrest increases serum potassium levels and induces acidaemia.

Eating disorders in male paediatric population is an increasing problem with asociated high morbidity, mortality and poor prognosis. This case highlights the importance of early intervention and support for affected individuals.

\section{G31(P) BIG HANDS HEALING LITTLE HEARTS}

${ }^{1}$ MJ Harvey, ${ }^{1} E$ Gracie, ${ }^{1} \mathrm{~L}$ Garvey, ${ }^{1} \mathrm{R}$ Fraser, ${ }^{4} \mathrm{~N}$ Cook, ${ }^{2} \mathrm{~B}$ Smith, ${ }^{3}$ WKE Peng, ${ }^{1} \mathrm{~N}$ Spenceley. ${ }^{1}$ Paediatric Critical Care, Royal Hospital for Children, Glasgow, UK; ${ }^{2}$ Department of Cardiology, Royal Hospital for Children, Glasgow, UK; ${ }^{3}$ Department of Cardiothoracic Surgery, Royal Hospital for Children, Glasgow, UK; ${ }^{4}$ Department of Cardiothoracic Surgery, Royal Brompton Hospital, London, UK

\subsection{6/archdischild-2018-rcpch.30}

Aims The Healing Little Hearts charity provides Cardiac Surgery and Critical Care for children with congenital heart disease in low and middle-income countries. We report the outcomes of the 5 th mission to the Hospital Universiti Sains Malaysia.

Methods A prospective, observational study in a Malaysian teaching hospital during a one week charity mission. The visiting charity team collaborated with local adult services to 
provide assessment, operative intervention and postoperative care to a paediatric cohort.

Results Twelve operations were undertaken for ten patients (table 1) with a median age of 3.4 years (IQR [2.3-5.8]) and weight $11.9 \mathrm{~kg}$ (IQR [9.1-14.5]). For those requiring cardiopulmonary bypass (CPB), median time was $107 \mathrm{~min}$ (IQR [92150]) and cross-clamp (XC) time $73 \mathrm{~min}$ (IQR [49.2-98.5]).

\begin{tabular}{llllll}
\multicolumn{7}{l}{ Abstract G31(P) Table 1 } & & & \\
\hline No. & $\begin{array}{l}\text { Age } \\
\text { (Years) }\end{array}$ & Procedure & $\begin{array}{l}\text { CPB } \\
\text { (Mins) }\end{array}$ & $\begin{array}{l}\text { XC } \\
\text { (Mins) }\end{array}$ & $\begin{array}{l}\text { Extubation Time } \\
\text { (Hours) }\end{array}$ \\
\hline 1 & 2 & VSD Closure & 60 & 34 & 2 \\
2 & 4.25 & REV Procedure (PA-VSD) & 320 & 226 & 58.08 \\
3 & 6.91 & AVSD Repair & 92 & 72 & 2.08 \\
4 & 0.02 & TAPVD Repair & 95 & 50 & 18.33 \\
5 & 6.33 & TOF Repair & 150 & 100 & 0.75 \\
6 & 3.08 & TOF Repair & 154 & 98 & 15.58 \\
7 & 2 & Partial PDA Occlusion & 0 & 0 & 3.75 \\
8 & 18.67 & Redo PV Implantation & 111 & 0 & 4.5 \\
9 & 3 & VSD Closure, PA de-banding and & 107 & 75 & 1 \\
& & reconstruction & & & \\
10 & 3.75 & VSD and PFO Closure & 71 & 47 & 1.25 \\
\hline
\end{tabular}

Fast-track extubation was performed in $70 \%$ with no reintubations. Post-operative morbidity was low and only patient 2 returned to theatre for additional procedures: delayed sternal closure and subsequent re-exploration. Case 3 was complicated by Junctional Ectopic Tachycardia, which resolved with medical management.

The team performed the institution's first redo surgery and neonatal operation. The local cardiac surgeon led his first paediatric VSD closure and Tetralogy of Fallot repair. All patients were extubated and discharged, or eligible for ICU discharge prior to conclusion of the mission.

Visiting PICU staff were resident 24 hours a day. The local ICU team were supported by twice daily PICU rounds, multi-disciplinary meetings and bedside teaching for nurses and trainees. The visiting Cardiologist undertook outpatient consultations, intra-and post-operative echocardiography and ECHO teaching. A morning lecture series delivered education to local trainees on topics including cardiac output, inotropes, heart failure and the surgical management of congenital heart disease.

Conclusions Healing Little Hearts provides cardiac surgical and critical care in developing countries. Short, well-planned missions can deliver quality care to a high turnover of patients with safe, early extubation and minimal morbidity, despite limited resources. Future missions will provide ongoing training and support the development of a safe, independent program capable of handling carefully selected paediatric congenital cardiac surgeries.

\section{G32(P) LATE PRETERM INFANTS AT GREATER RISK OF PAEDIATRIC INTENSIVE CARE ADMISSION AND LONGER INVASIVE VENTILATION DUE TO RESPIRATORY SYNCITIAL VIRUS BRONCHIOLITIS}

S Followell, M Christopherson, P Davis. Paediatric Intensive Care, Bristol Royal Hospital for Children, Bristol, UK

10.1136/archdischild-2018-rcpch.31
Aims Only a small proportion of late-preterm (LPT) infants (34-36+6 weeks gestational age) require admission to NICU for respiratory support at birth. We aimed to evaluate the subsequent impact of Respiratory Syncitial Virus (RSV) on LPT infants compared to FT infants.

Methods A retrospective cohort study of infants admitted to a regional tertiary PICU with a primary diagnosis of RSV-positive bronchiolitis over a five-year period was undertaken. LPT infants were compared to FT infants (37 weeks and greater gestational age). The relative risk of PICU admission for RSVpositive bronchiolitis for LPT and FT infants in our regional population was calculated using Office of National Statistics data. Ventilation rates between the 2 groups admitted to PICU were compared using Chi-squared. Non-parametric statistical analysis was undertaken to identify any significant differences in other resource utilisation between the LPT and FT infants.

Results 40 LPT and 102 FT infants were admitted to PICU with a diagnosis of RSV-positive bronchiolitis from 2012 to 2017. Of infants born between 34 and 42 weeks in this period, 4.4\% were LPT. However, LPT infants constituted $28.2 \%$ of the study population admitted to PICU with RSVpositive bronchiolitis ( $\mathrm{RR}=8.44, \quad 95 \% \mathrm{CI}$ 5.85-12.15, $\mathrm{p}<0.0001)$. Invasive ventilation rates were similar between LPT and FT infants (75\% LPT vs. $68.6 \%$ FT, $\chi 2=0.08$, DF1, $\mathrm{p}=0.78$ ). The median length of invasive ventilation was 6 days (IQR 5-11) among LPT infants and 5 days (IQR 4-7) for FT infants $(\mathrm{W}=1322 ; \mathrm{p}=0.039)$. The median length of PICU stay was 7 days (IQR 5-12) for LPT infants and 6 days (IQR 4-8) among FT infants $(\mathrm{W}=2574 ; \mathrm{p}=0.015)$. There were no PICU deaths among the 142 RSV-positive bronchiolitis admissions.

Conclusion Despite few LPT infants requiring respiratory input from a neonatologist at birth, following discharge home, they are at significantly increased risk of admission to PICU with RSV-positive bronchiolitis, compared to FT infants, with longer periods of invasive ventilation and lengthier admissions. This may reflect the importance of reaching full term for lung development and the control of breathing, especially when exposed to common respiratory viruses during infancy.

\section{G33(P) OVERVIEW OF RARE RENAL DISEASES AT A PAEDIATRIC RENAL CENTRE THROUGH THE NATIONAL REGISTRY OF RARE KIDNEY DISEASES (RADAR) IN THE UNITED KINGDOM}

'L Kerecuk, 'M Kokocinska, 'S Parkes, ${ }^{1} \mathrm{~J}$ Kainth, ${ }^{2} \mathrm{M}$ Dillon. 'Nephrology, Birmingham Children's Hospital, Birmingham, UK; ${ }^{2}$ National Registry of Rare Kidney Diseases (RADAR), UK Renal Registry, Bristol, UK

\subsection{6/archdischild-2018-rcpch.32}

Aims Rare kidney diseases need better understanding and the starting point is collection of clinical data. Therefore our centre is participating in RaDaR which is a UK Renal Association initiative designed to gather information. RaDaR recruitment began in 2010 and now covers more than 40 conditions. There are around 10000 recruits from 78 renal adult and paediatric units in the UK. Our Paediatric Renal Centre is the leading recruiting hospital in the UK.

To describe the range of range of conditions and patient numbers recruited to RADAR at $\mathrm{BCH}$ which is a national tertiary renal referral hospital in the UK.

Methods The RaDaR dataset is defined by the UK Renal Registry in association with the Rare Disease Groups, made 